\title{
LEGENDRIAN LENS SPACE SURGERIES
}

\author{
HANSJÖRG GEIGES AND SINEM ONARAN
}

\begin{abstract}
We show that every tight contact structure on any of the lens spaces $L\left(n s^{2}-s+1, s^{2}\right)$ with $n \geq 2, s \geq 1$, can be obtained by a single Legendrian surgery along a suitable Legendrian realisation of the negative torus knot $T(s,-(s n-1))$ in the tight or an overtwisted contact structure on the 3-sphere.
\end{abstract}

\section{INTRODUCTION}

A knot $K$ in the 3 -sphere $S^{3}$ is said to admit a lens space surgery if, for some rational number $r$, the 3-manifold obtained by Dehn surgery along $K$ with surgery coefficient $r$ is a lens space. In [17] L. Moser showed that all torus knots admit lens space surgeries. More precisely, $-(a b \pm 1)$-surgery along the negative torus knot $T(a,-b)$ results in the lens space $L\left(a b \pm 1, a^{2}\right)$, cf. [21; for positive torus knots one takes the mirror of the knot and the surgery coefficient of opposite sign, resulting in a negatively oriented lens space. Contrary to what was conjectured by Moser, there are surgeries along other knots that produce lens spaces. The first example was due to J. Bailey and D. Rolfsen [1, who constructed the lens space $L(23,7)$ by integral surgery along an iterated cable knot.

The question which knots admit lens space surgeries is still open and the subject of much current research. The fundamental result in this area is due to CullerGordon-Luecke-Shalen [2], proved as a corollary of their cyclic surgery theorem: if $K$ is not a torus knot, then at most two surgery coefficients, which must be successive integers, can correspond to a lens space surgery. For more recent work, relating this question to Floer theory, see [12, 14, 18, 20, for instance.

The converse question, which lens spaces can be obtained by a single surgery on the 3 -sphere, is of course trivial in the topological setting: the lens space $L(p, q)$, as an oriented manifold, is the result of a $(-p / q)$-surgery along the unknot.

In the present note we consider this converse question for contact manifolds: which tight contact structures on a given lens space can be obtained by a single contact (-1)-surgery (also known as Legendrian surgery) along a Legendrian knot in $S^{3}$ with some contact structure? Here the topological restrictions become relevant in the search for contact structures on lens spaces that cannot be constructed in this way.

J. Rasmussen proved in [20, Corollary 4] that the only integral surgery on $S^{3}$ that produces the lens space $L(4 m+3,4)$ is surgery along the negative torus knot $T(2,-(2 m+1))$ with coefficient $-(4 m+3)$. (The statement about the surgery coefficient is not contained in 20, but this follows immediately from [17, because other surgery coefficients lead to a different lens space or a Seifert manifold with three

2010 Mathematics Subject Classification. 53D35; 53D10, 57M25, 57R65. 
multiple fibres.) Beware that Rasmussen uses the opposite orientation convention for lens spaces.

Based on Rasmussen's result, O. Plamenevskaya claimed in [19, Proposition 5.4] that only one of the three, up to isotopy, (positive) tight contact structures on $L(7,4)$ can be obtained via Legendrian surgery on some contact structure on $S^{3}$. This assertion, as we shall see, is erroneous. The main result of this note is the following.

Theorem 1.1. For any pair of integers $n \geq 2, s \geq 1$, every tight contact structure on the lens space $L\left(n s^{2}-s+1, s^{2}\right)$ can be obtained by a single Legendrian surgery along a suitable Legendrian realisation of the negative torus knot $T(s,-(s n-1))$ in some contact structure on $S^{3}$.

Remark 1.2. (1) On the lens space $L\left(n s^{2}-s+1, s^{2}\right)$ there are, for $s \geq 2$, precisely $(s+1)(n-1)$ distinct tight contact structures up to isotopy; for $s=1$ the number is $n-1$. (One reason why we restrict attention to these lens spaces is that the arithmetic for determining the number of tight structures is simple.) As we shall be realising these structures by different contact surgery diagrams, it may not seem to be clear how to distinguish non-isotopic but diffeomorphic structures. However, those different contact surgery diagrams will correspond to the same topological surgery diagram, and this fixes the resulting manifold, so that the notion of isotopy becomes meaningful.

(2) There is a more fundamental reason for considering only the lens spaces $L\left(n s^{2}-s+1, s^{2}\right)$ : a major share of the tight contact structures stem from exceptional realisations of the torus knot $T(s,-(s n-1))$ (i.e. as Legendrian knots $L \subset\left(S^{3}, \xi_{\text {ot }}\right)$ in an overtwisted contact structure with $\left.\xi_{\text {ot }}\right|_{S^{3} \backslash L}$ tight), and systematically we can only produce them for those particular torus knots. One can expect that the lens spaces $L\left(n s^{2}+s+1, s^{2}\right)$, coming from surgery along the torus knots $T(s,-(s n+1))$, may be treated in the same fashion.

(3) The maximal Thurston-Bennequin number of Legendrian realisations of the negative torus knot $T(a,-b)$ in the tight $\left(S^{3}, \xi_{\text {st }}\right)$ equals $-a b$, so the maximal topological surgery coefficient for a Legendrian surgery is $-(a b+1)$. It seems reasonable to conjecture that the theorem holds true for all lens spaces $L\left(a b+1, a^{2}\right)$.

We assume that the reader is familiar with the elements of contact topology on the level of [7]. In particular, our argument depends on the presentation of contact 3 -manifolds in terms of contact $( \pm 1)$-surgery diagrams, see [4] and [7, Section 6.4].

\section{Contact structures on Lens Spaces}

As explained in the introduction, lens spaces come with a natural orientation. When we speak of a 'contact structure' $\xi$ on an oriented 3-manifold, we always mean a positive, oriented contact structure, i.e. $\xi=\operatorname{ker} \alpha$ with $\alpha \wedge \mathrm{d} \alpha>0$, and $\alpha$ is given up to multiplication by a positive function.

The number of tight contact structures on lens spaces has been determined independently by E. Giroux 9] and K. Honda [13].

Theorem 2.1 (Giroux, Honda). On the lens space $L(p, q)$ with $p>q>0$ and $\operatorname{gcd}(p, q)=1$, the number of tight contact structures is given by

$$
\left(a_{0}-1\right) \cdots\left(a_{k}-1\right),
$$


where the $a_{i} \geq 2$ are the terms in the negative continued fraction expansion

$$
\frac{p}{q}=a_{0}-\frac{1}{a_{1}-\frac{1}{a_{2}-\cdots-\frac{1}{a_{k}}}}=:\left[a_{0}, \ldots, a_{k}\right] .
$$

For our family of lens spaces, this number is easy to compute.

Corollary 2.2. The number of tight contact structures on $L\left(n s^{2}-s+1, s^{2}\right)$ is $n-1$ for $s=1$, and $(s+1)(n-1)$ for $s \geq 2$.

Proof. The case $s=1$ is clear. For $s \geq 2$, we claim that

$$
\frac{n s^{2}-s+1}{s^{2}}=[n, s+2, \underbrace{2, \ldots, 2}_{s-2}] .
$$

The result then follows from Theorem 2.1.

Inductively one sees that

$$
[\underbrace{2, \ldots, 2}_{s-2}]=\frac{s-1}{s-2} \text {. }
$$

Then

$$
\begin{aligned}
{[n, s+2, \underbrace{2, \ldots, 2}_{s-2}] } & =n-\frac{1}{s+2-\frac{1}{[2, \ldots, 2]}} \\
& =\frac{n s^{2}-s+1}{s^{2}},
\end{aligned}
$$

as was claimed.

Our aim is to find Legendrian realisations of the torus $\operatorname{knot} T(s,-(s n-1))$ in some contact structure on $S^{3}$ such that Legendrian surgery on the knot produces a tight contact structure on the lens space $L\left(n s^{2}-s+1, s^{2}\right)$. This requires, first of all, that the Thurston-Bennequin invariant $\mathrm{tb}$ of these realisations equals $-s(s n-1)$, so that topologically we perform a surgery with framing $-\left(n s^{2}-s+1\right)$. Secondly, a necessary condition for the contact structure on the surgered manifold to be tight is that we start with the standard tight contact structure $\xi_{\text {st }}$ on $S^{3}$, or with an exceptional realisation of $T(s,-(s n-1))$ in an overtwisted contact structure; see Remark 1.2 (2) for the definition of exceptional Legendrian knots - these are also referred to as nonloose Legendrian knots.

We shall be representing $T(s,-(s n-1))$ as a Legendrian knot $L$ in a contact surgery diagram of some contact structure on $S^{3}$. One will then see directly that Legendrian surgery on this knot produces a contact surgery diagram for a tight contact structure on $L\left(n s^{2}-s+1, s^{2}\right)$. Thus, with hindsight the contact structure on the complement of $L$ was tight.

In order to distinguish the contact structures obtained in this fashion, we need two well-known homotopical invariants of tangent 2-plane fields on 3-manifolds. The first is the Euler class, which modulo 2-torsion detects homotopy over the 2 -skeleton; the second is the so-called $d_{3}$-invariant. 
Example 2.3. The Euler class suffices to settle the case $s=1$ of the theorem. In $\left(S^{3}, \xi_{\text {st }}\right)$ there are $n-1$ Legendrian realisations of the (oriented) unknot with $\mathrm{tb}=-n+1$, with rotation numbers in the range

$$
\text { rot } \in\{-n+2,-n+4, \ldots, n-4, n-2\},
$$

given by adding $n-2$ zigzags, distributed on the left and the right, to the standard front projection picture of an unknot with $t b=-1$ and rot $=0$. Legendrian surgery on these knots produces the lens space $L(n, 1)$. The almost complex structure on the corresponding symplectic handlebody $X$, which has cohomology $H^{2}(X) \cong \mathbb{Z}$, has first Chern class equal to rot, and by [15. Theorem 1.2], this distinguishes the $n-1$ contact structures on $L(n, 1)$ up to isotopy.

Recall from [10, Section 4] that one can associate with any oriented tangent 2plane field $\eta$ on a closed, orientable 3 -manifold $Y$ a homotopy invariant $d_{3}(\eta) \in \mathbb{Q}$, provided the Euler class $e(\eta)$ is a torsion class. This is the homotopy obstruction over the 3-skeleton of $Y$ in the sense that two such 2-plane fields that are homotopic over the 2-skeleton are homotopic over $Y$ if and only if they have the same $d_{3^{-}}$ invariant.

Suppose the contact manifold $(Y, \xi)$ is given in terms of a surgery presentation $\mathbb{L}=\mathbb{L}_{+} \sqcup \mathbb{L}_{-} \subset\left(S^{3}, \xi_{\text {st }}\right)$, i.e. $\mathbb{L}$ is a Legendrian link, and $(Y, \xi)$ is the result of performing contact $( \pm 1)$-surgery along the components of the sublinks $\mathbb{L}_{ \pm}$. In this situation, the $d_{3}$-invariant can be computed as follows, see [4, Corollary 3.6], where $X$ is the 4-dimensional handlebody determined by the surgery description, $\chi(X)$ its Euler characteristic, and $\sigma(X)$ its signature.

Proposition 2.4. Suppose that the Euler class $e(\xi)$ is torsion, and $\operatorname{tb}\left(L_{i}\right) \neq 0$ for each $L_{i} \in \mathbb{L}_{+}$. Then

$$
d_{3}(\xi)=\frac{1}{4}\left(c^{2}-3 \sigma(X)-2 \chi(X)\right)+q,
$$

where $q$ denotes the number of components of $\mathbb{L}_{+}$, and $c \in H^{2}(X)$ is the cohomology class determined by $c\left(\Sigma_{i}\right)=\operatorname{rot}\left(L_{i}\right)$ for each $L_{i} \subset \mathbb{L}$.

For the computation of the rational number $c^{2}$, write $M$ for the linking matrix of the link $\mathbb{L}$, with diagonal entries given by the topological surgery framings. Let rot be the vector of rotation numbers of the link components. Solve the linear system $M \mathbf{x}=\underline{\text { rot }}$ over $\mathbb{Q}$. Then $c^{2}=\mathrm{x}^{\mathrm{t}} M \mathbf{x}$.

\section{The LENS SPACE $L(7,4)$}

In order to see where the error occurs in [19, we begin with Plamenevskaya's example $L(7,4)$, i.e. the case $n=2, s=2$. This lens space admits three tight contact structures.

According to Rasmussen's result cited in the introduction, the only way to obtain $L(7,4)$ by an integral surgery on $S^{3}$ is a $(-7)$-surgery along a left-handed trefoil knot $T(2,-3)$. We therefore need to look for Legendrian realisations of the lefthanded trefoil with $\mathrm{tb}=-6$ either in $\left(S^{3}, \xi_{\text {st }}\right)$ or as an exceptional knot in an overtwisted contact structure on $S^{3}$.

By [6. Section 4.1], -6 is the maximal Thurston-Bennequin number for Legendrian realisations of the left-handed trefoil knot in $\left(S^{3}, \xi_{\mathrm{st}}\right)$, and there are precisely two realisations (as oriented Legendrian knots) with this maximal tb, distinguished by their rotation numbers rot $= \pm 1$. They are shown in Figure 1 . 

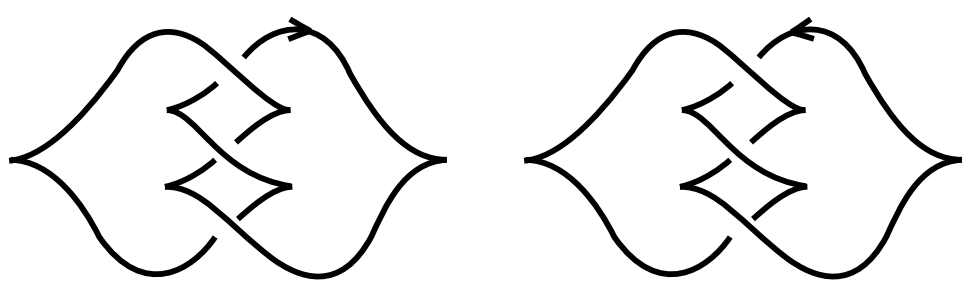

Figure 1. The left-handed trefoils in $\left(S^{3}, \xi_{\text {st }}\right)$ with $\mathrm{tb}=-6$.

An exceptional realisation $L \subset S^{3}$ of the left-handed trefoil is shown in Figure 2 We shall check in a moment that $\operatorname{tb}(L)=-6$; Legendrian surgery along $L$ then produces $L(7,4)$.

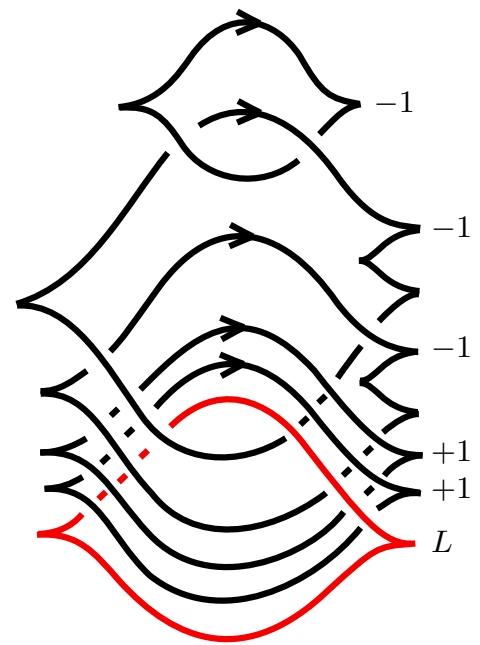

Figure 2. An exceptional left-handed trefoil $L$ with $\mathrm{tb}=-6$.

The Kirby moves to verify the topological part of this statement are shown in Figures 3 and 4 The latter shows how to separate $L$ from the surgery link by $1+2$ handle slides (where in the first step we slide both strands simultaneously), turning it into a left-handed trefoil in $S^{3}$. The two-component surgery link does indeed represent the 3 -sphere, since surgery along the 0 -framed meridian cancels the (-2)-surgery; for instance, one can use a slam-dunk [11, Figure 5.30].

To see that $L$ is exceptional, observe that - by the cancellation lemma [3], cf. [7. Proposition 6.4.5] - contact (-1)-surgery along $L$ in Figure 2 cancels one of the contact $(+1)$-surgeries. Then the remaining surgery diagram contains only a single contact $(+1)$-surgery along a standard Legendrian unknot, which by 4 produces the unique tight (and Stein fillable) contact structure on $S^{1} \times S^{2}$, and the further contact $(-1)$-surgeries then produce a Stein fillable and hence tight contact structure.

On the other hand, the contact structure on $S^{3}$ given by the surgery diagram in Figure 2 (without any surgery along $L$ ) is overtwisted, since its $d_{3}$-invariant is $3 / 2$ (recall that $d_{3}\left(\xi_{\mathrm{st}}\right)=-1 / 2$ ). Indeed, the linking matrix (ordering the knots from 

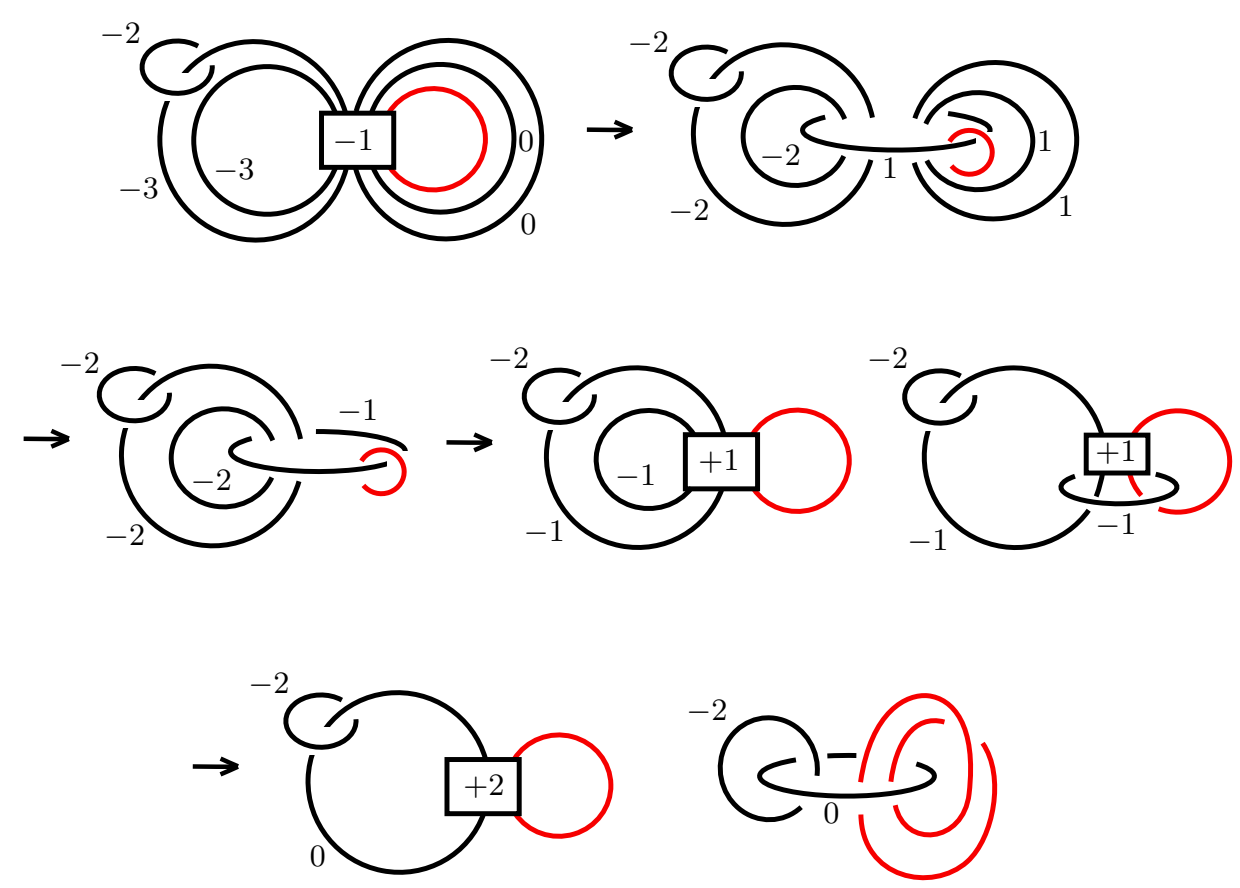

Figure 3. Kirby moves for Figure 2

bottom to top) is

$$
M=\left(\begin{array}{ccccc}
0 & -1 & -1 & -1 & 0 \\
-1 & 0 & -1 & -1 & 0 \\
-1 & -1 & -3 & -1 & 0 \\
-1 & -1 & -1 & -3 & -1 \\
0 & 0 & 0 & -1 & -2
\end{array}\right)
$$

with signature $\sigma=-1$ (instead of computing $\sigma$ from the matrix one can see this from the Kirby moves in Figure 3 by keeping track of the blow-ups and blowdowns). Since we are adding five 2-handles to the 4-ball, the Euler characteristic of the handlebody is $\chi=6$. The vector of rotation numbers is $\underline{\text { rot }}=(0,0,1,1,0)^{\mathrm{t}}$, and the solution of $M \mathbf{x}=\underline{\text { rot }}$ is $\mathbf{x}=(-7,-7,3,4,-2)^{\mathrm{t}}$. This gives $c^{2}=\mathbf{x}^{\mathrm{t}} M \mathbf{x}=7$, and hence $d_{3}=3 / 2$ with formula (11).

We still need to check that $\operatorname{tb}(L)=-6$. For this we may use the formula from [16. Lemma 6.6], cf. [8, Lemma 3.1] and [5. Consider the extended linking matrix

$$
M_{0}=\left(\begin{array}{cccccc}
0 & -1 & -1 & -1 & -1 & 0 \\
-1 & 0 & -1 & -1 & -1 & 0 \\
-1 & -1 & 0 & -1 & -1 & 0 \\
-1 & -1 & -1 & -3 & -1 & 0 \\
-1 & -1 & -1 & -1 & -3 & -1 \\
0 & 0 & 0 & 0 & -1 & -2
\end{array}\right)
$$

which now includes $L$ as the first link component, with the first diagonal entry set to zero. Write $\mathrm{tb}_{0}$ for the Thurston-Bennequin invariant of $L$ as a knot in the unsurgered copy of $S^{3}$, i.e. here $\mathrm{tb}_{0}=-1$. Then, in the surgered manifold (which 


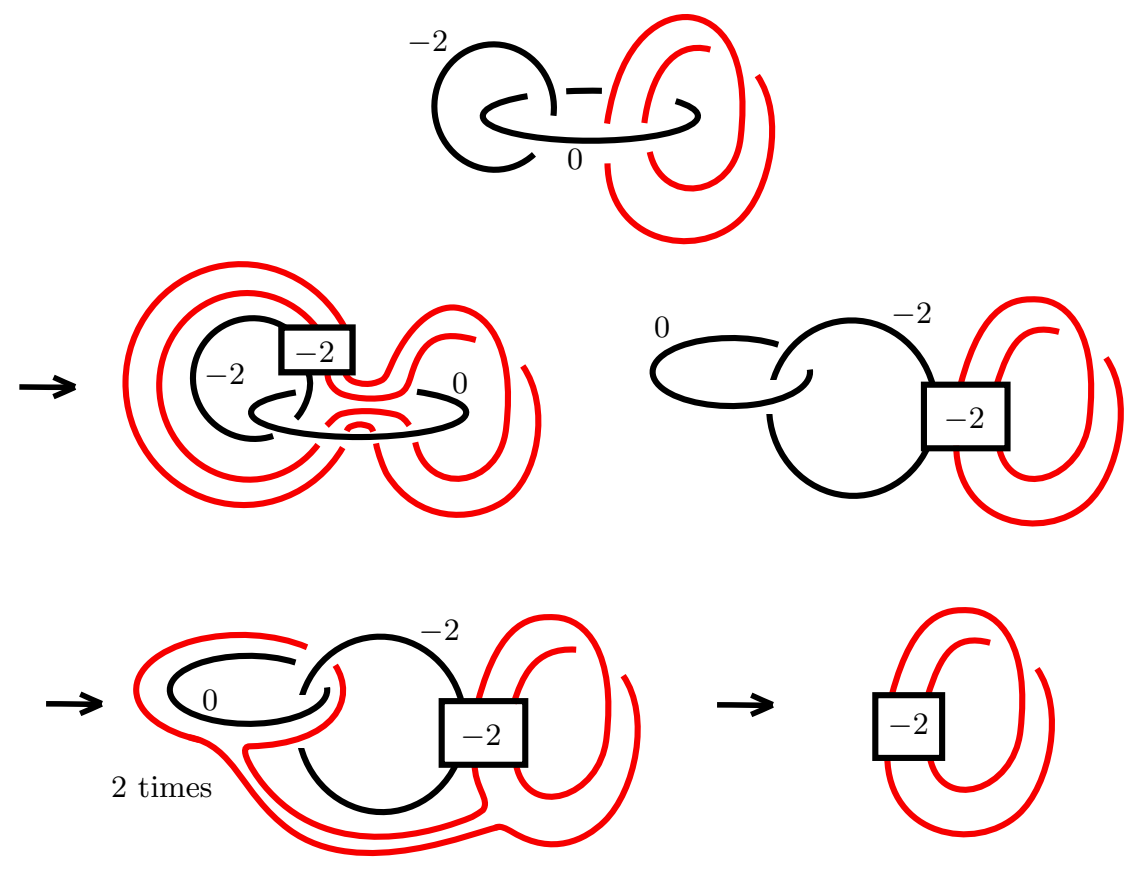

Figure 4. The final handle slides.

here is another copy of $S^{3}$ ) one has

$$
\operatorname{tb}(L)=\mathrm{tb}_{0}+\frac{\operatorname{det} M_{0}}{\operatorname{det} M}=-1+\frac{5}{-1}=-6 .
$$

Remark 3.1. Alternatively, one can determine $\operatorname{tb}(L)$ by keeping track of the framing of $L$ during the Kirby moves. Start with a Legendrian push-off $L^{\prime}$ of $L$ in the original diagram, which has linking -1 with $L$. In the last diagram of Figure 3 . we then have linking number $1 \mathrm{k}\left(L, L^{\prime}\right)=2$ (as knots in the unsurgered $S^{3}$ ). After the handle slides in Figure 4 , the parallel knot $L^{\prime}$ will likewise pass twice through the $(-2)$-box, so two strands of $L^{\prime}$ will each receive a $(-2)$-twisting relative to two strands of $L$, resulting in $\operatorname{tb}(L)=2-2^{3}=-6$.

Proposition 3.2. Legendrian surgery along the three left-handed trefoil knots in Figures 1 and 2 produces the three tight contact structures on $L(7,4)$.

Remark 3.3. A word of clarification is in order. The result of a surgery along a knot does not depend on the orientation of the knot. In what sense, then, can the two knots in Figure 1 be said to correspond to two non-isotopic contact structures on a given copy of the lens space $L(7,4)$ ?

In an integral surgery diagram, read as a Kirby diagram for a 4-dimensional handlebody $X$, a choice of orientation on a knot $K$ amounts to a choice of positive generator in the corresponding $\mathbb{Z}$-summand of $H_{2}(X)$, represented by an oriented Seifert surface for $K$ glued with the core disc in the handle.

The left-handed trefoil, like all torus knots, is a reversible knot, i.e. it is isotopic to itself with reversed orientation. This means that the two oriented knots in Figure 1 are topologically isotopic. The time- 1 map of this isotopy gives us an identification 
of the two handlebodies such that either knot corresponds to the positive generator of $H_{2}(X)$. It is with respect to this identification that we compare the resulting contact structures; likewise for the contact structure coming from Figure 2.

The same comments apply to the general case discussed in the subsequent sections. In particular, once an orientation of the torus knot has been fixed, we can use the Euler class to distinguish contact structures on the lens space resulting from surgery, without any need to consider the action of diffeomorphisms on the second cohomology group.

Proof of Proposition 3.2. First of all, observe that Legendrian surgery along the examples in Figure 1 produces Stein fillable and hence tight contact structures on $L(7,4)$. Tightness of the contact structure obtained by surgery along the exceptional left-handed trefoil in Figure 2 was explained above; this was our argument for seeing that the Legendrian knot $L$ is indeed exceptional.

For the examples in Figure 1 we compute $d_{3}=-2 / 7$ from $M=(-7), \chi=2$, $\sigma=-1$, and $c^{2}=-1 / 7$. By [10, Proposition 2.3], the first Chern class of the Stein surface $(X, J)$ described by the respective diagram evaluates to rot $= \pm 1$ on the positive generator of $H_{2}(X)$. The Euler class of the respective contact structure induced on the boundary $\partial X=L(7,4)$ then equals $\pm 1 \in H^{2}(L(7,4))=\mathbb{Z}_{7}$, so the two contact structures are non-isotopic.

For the example in Figure 2, Legendrian surgery cancels one of the contact $(+1)$-surgeries. The remaining diagram has linking matrix (ordering the knots from bottom to top)

$$
M=\left(\begin{array}{cccc}
0 & -1 & -1 & 0 \\
-1 & -3 & -1 & 0 \\
-1 & -1 & -3 & -1 \\
0 & 0 & -1 & -2
\end{array}\right)
$$

with signature $\sigma=-2$. The Euler characteristic of the handlebody is $\chi=5$, and the vector of rotation numbers is $\underline{\text { rot }}=(0,1,1,0)^{\mathrm{t}}$. The solution of $M \mathbf{x}=\underline{\text { rot }}$ is given by $\mathbf{x}=(-1,0,0,0)^{\mathrm{t}}$, hence $c^{2}=\mathbf{x}^{\mathrm{t}} M \mathbf{x}=0$, and $d_{3}=(0+6-10) / 4+\overline{1=}$, which distinguishes this contact structure from the other two.

Remark 3.4. For Legendrian surgery diagrams of the three tight structures on $L(7,4)$ involving two-component links see [19, Figure 6]. The error in [19] occurs in the computation of the $d_{3}$-invariant for the contact structure coming from the examples in Figure 1 Plamenevskaya obtains $d_{3}=0$ and then argues, correctly, that the two structures with $d_{3}=-2 / 7$ cannot come from an exceptional trefoil.

\section{The Lens Spaces $L(4 m+3,4)$}

As a first generalisation, we now prove Theorem 1.1 for $s=2$ but arbitrary $n$. Here we shall distinguish the contact structures by their Euler classes. For notational convenience, we set $m=n-1$, so instead of $L(4 n-1,4)$ we consider $L(4 m+3,4)$ with $m \geq 1$.

The lens space $L(4 m+3,4)$ is obtained by - $(4 m+3)$-surgery on the torus knot $T(2,-(2 m+1))$, so we need to find Legendrian realisations of this torus knot with $\mathrm{tb}=-(4 m+2)$.

The following result is due to Etnyre-Honda [6, Section 4.1], where the reader can also find explicit front projection diagrams of the knots in question. 
Proposition 4.1 (Etnyre-Honda). The maximal Thurston-Bennequin invariant of Legendrian realisations of $T(2,-(2 m+1))$ in $\left(S^{3}, \xi_{\mathrm{st}}\right)$ is $-(4 m+2)$. Up to Legendrian isotopy, there are $2 m$ realisations with this maximal $\mathrm{tb}$, with rotation number in the range

$$
\operatorname{rot} \in\{-2 m+1,-2 m+3, \ldots, 2 m-3,2 m-1\} .
$$

Legendrian surgery on these knots yields $2 m$ tight contact structures on the lens space $L(4 m+3,4)$, distinguished by their Euler class in $H^{2}(L(4 m+3,4))=\mathbb{Z}_{4 m+3}$, which, as above, is given by the reduction of the rotation number modulo $4 m+3$.

The remaining $m$ tight contact structures on $L(4 m+3,4)$ have to come from exceptional realisations of the torus knot $T(2,-(2 m+1))$ in some overtwisted contact structure on $S^{3}$.

Proposition 4.2. For $(k, l) \in \mathbb{N}_{0} \times \mathbb{N}_{0}$ with $k+l=m-1$, the Legendrian knot $L$ shown in the contact surgery diagram of Figure 5 is an exceptional realisation of $T(2,-(2 m+1))$ in $S^{3}$ with $\mathrm{tb}=-(4 m+2)$.

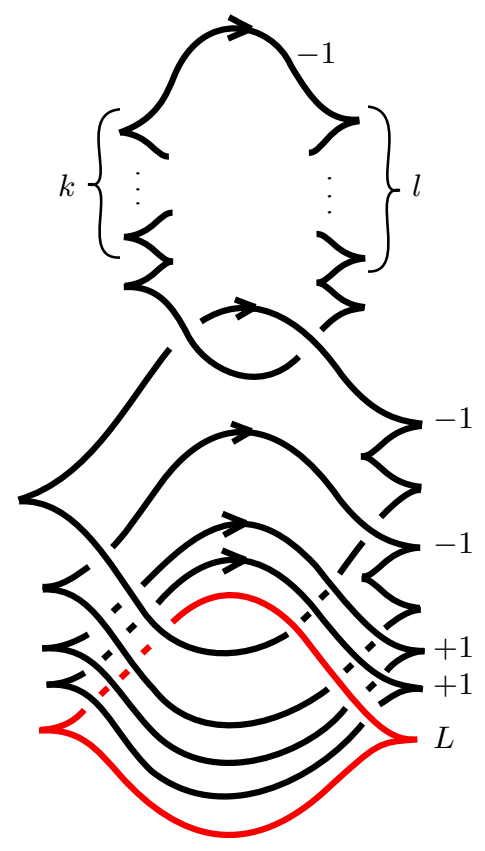

FiguRE 5. Exceptional realisations of $T(2,-(2 m+1)), m=k+l+1$.

Remark 4.3. Exceptional realisations of the torus knots $T(2,-(2 m+1))$ have previously been described in [16]. The Kirby moves in Figure 3 are those of [16, Figure 18]. However, the purported exceptional realisations in [16, Figure 17] do not actually correspond to this Kirby diagram.

Proof of Proposition 4.2. The Kirby moves in Figures 3 and 4 once again confirm the topological part of the statement. Simply replace the (-2)-framing in the first diagram of Figure 3 by $-(m+1)=-n$, which remains unchanged throughout the moves, and instead of the $1+2$ slides in Figure 4 perform $1+n$ slides. Likewise, 
the argument that surgery along $L$ in Figure 5 produces a tight contact structure is as before.

For the computation of $\operatorname{tb}(L)$ in the surgered $S^{3}$, observe that only the last diagonal entry -2 in the matrices $M$ and $M_{0}$ (before Proposition 3.2) needs to be replaced by $-(m+1)$. By expanding the respective determinants along the last row, one finds that $\operatorname{det} M=-1$ remains unchanged, and $\operatorname{det} M_{0}=-3+4(m+1)=$ $4 m+1$. This gives $\operatorname{tb}(L)=-(4 m+2)$, as desired.

Next we compute the $d_{3}$-invariant of the surgered $S^{3}$. As before we have $\sigma=-1$ and $\chi=6$. We have

$$
\underline{\mathrm{rot}}=(0,0,1,1, l-k)^{\mathrm{t}},
$$

and the solution of $M \mathbf{x}=\underline{\text { rot }}$ is given by

$$
\mathbf{x}=(-6 k-2 l-7,-6 k-2 l-7,3 k+l+3,3 k+l+4,-2)^{\mathrm{t}} .
$$

It follows that $d_{3}=2 k+3 / 2$, so the contact structure is overtwisted, and $L$ is exceptional.

The following proposition shows that Legendrian surgery along these exceptional knots produces the required $m$ tight structures on $L(4 m+3,4)$, distinct from the $2 m$ we found previously.

Proposition 4.4. Legendrian surgery along the knot $L$ shown in Figure 5 (for the various choices of $k, l)$ produces $m$ tight contact structures on $L(4 m+3,4)$ with Euler class in $H^{2}(L(4 m+3,4))=\mathbb{Z}_{4 m+3}$ in the range

$$
-2 m+2,-2 m+6, \ldots, 2 m-6,2 m-2 \bmod 4 m+3 .
$$

Proof. We compute the Euler class of the contact structures on $L(4 m+3,4)$ obtained via Legendrian surgery along these exceptional knots. To this end, we need to compute the rotation number of $L$ as a knot in the overtwisted $S^{3}$ obtained by surgery along the link in Figure 5. According to [16, Lemma 6.6], cf. [8, Lemma 3.1], this rotation number is given by

$$
\operatorname{rot}(L)=\operatorname{rot}_{0}-\left\langle\underline{\operatorname{rot}}, M^{-1} \underline{1 \mathrm{k}}\right\rangle,
$$

where rot $_{0}$ denotes the rotation number of $L$ in the unsurgered copy of $S^{3}$ (here $\operatorname{rot}_{0}=0$ ), and $\underline{\mathrm{k}}$ is the vector of linking numbers of $L$ with the components of the surgery link. We give $L$ the clockwise orientation in the diagram, then

$$
\underline{\mathrm{lk}}=(-1,-1,-1,-1,0)^{\mathrm{t}}
$$

and

$$
M^{-1} \underline{\underline{\mathrm{k}}}=(-4 m-2,-4 m-2,2 m+1,2 m+2,-2)^{\mathrm{t}} .
$$

This yields

$$
\operatorname{rot}(L)=-(4 m+3)+2(l-k) \equiv 2(l-k) \bmod 4 m+3 .
$$

Hence, modulo $4 m+3$, the rotation number can take on $m$ distinct values in the range

$$
\operatorname{rot} \in\{-2 m+2,-2 m+6, \ldots, 2 m-6,2 m-2\} .
$$

Here is an alternative way to compute the Euler class of the contact structure on $L(4 m+3,4)$ obtained by surgery on the link in Figure 5 (including a contact $(-1)$ surgery along $L$ ), which is more direct than computing $\operatorname{rot}(L)$ in the surgered $S^{3}$.

Write $X$ for the 4-dimensional handlebody described by this diagram, so that $\partial X=L(4 m+3,4)$. The first homology group $H_{1}(\partial X)=\mathbb{Z}_{4 m+3}$ is generated by 
the classes of the meridians $\left[\mu_{L}\right],\left[\mu_{1}\right], \ldots,\left[\mu_{5}\right]$, and the relations are given by the linking matrix, cf. 4. For ease of notation, we change all signs in that matrix:

$$
\left(\begin{array}{cccccc}
2 & 1 & 1 & 1 & 1 & 0 \\
1 & 0 & 1 & 1 & 1 & 0 \\
1 & 1 & 0 & 1 & 1 & 0 \\
1 & 1 & 1 & 3 & 1 & 0 \\
1 & 1 & 1 & 1 & 3 & 1 \\
0 & 0 & 0 & 0 & 1 & m+1
\end{array}\right) \cdot\left(\begin{array}{l}
{\left[\mu_{L}\right]} \\
{\left[\mu_{1}\right]} \\
{\left[\mu_{2}\right]} \\
{\left[\mu_{3}\right]} \\
{\left[\mu_{4}\right]} \\
{\left[\mu_{5}\right]}
\end{array}\right)=\left(\begin{array}{l}
0 \\
0 \\
0 \\
0 \\
0 \\
0
\end{array}\right) .
$$

This yields the relations

$$
(4 m+3)\left[\mu_{4}\right]=0, \quad-\left[\mu_{L}\right]=\left[\mu_{1}\right]=\left[\mu_{2}\right]=2\left[\mu_{4}\right], \quad\left[\mu_{3}\right]=-\left[\mu_{4}\right], \quad\left[\mu_{5}\right]=-4\left[\mu_{4}\right],
$$

so that indeed $H_{1}(\partial X)=\mathbb{Z}_{4 m+3}$, generated by the class $\left[\mu_{4}\right]$. The class $\left[\mu_{L}\right]$ can likewise be taken as a generator, since $-2(m+1)\left[\mu_{L}\right]=(4 m+4)\left[\mu_{4}\right]=\left[\mu_{4}\right]$.

Now, as discussed in [4, the Poincaré dual of the Euler class $e(\xi)$ of the contact structure on $\partial X$ is given by the vector of rotation numbers of the link components, expressed in terms of the classes of meridians, that is,

$$
e(\xi)=\left[\mu_{3}\right]+\left[\mu_{4}\right]+(l-k)\left[\mu_{5}\right]=2(l-k)\left[\mu_{L}\right],
$$

which confirms the calculation in the foregoing proof.

$$
\text { 5. The Lens Spaces } L\left(n s^{2}-s+1, s^{2}\right)
$$

Finally, we deal with the general case $n \geq 2, s \geq 2$ of Theorem 1.1. The relevant result from 6] can now be phrased as follows.

Proposition 5.1 (Etnyre-Honda). The maximal Thurston-Bennequin invariant of Legendrian realisations of $T(s,-(s n-1))$ in $\left(S^{3}, \xi_{\mathrm{st}}\right)$ is $-s(s n-1)$. Up to Legendrian isotopy, there are $2(n-1)$ realisations with this maximal tb, with rotation number in the range

$$
\{-(n-1) s+1,-(n-3) s \pm 1, \ldots,(n-3) s \pm 1,(n-1) s-1\} .
$$

As in the case $s=2$, Legendrian surgery along these knots gives us 2( $n-1)$ tight contact structures on $L\left(n s^{2}-s+1, s^{2}\right)$. It remains to find $(s-1)(n-1)$ exceptional realisations of $T(s,-(s n-1))$ in $S^{3}$ that will gives us the remaining tight structures on the lens space.

Proposition 5.2. For $(k, l) \in \mathbb{N}_{0} \times \mathbb{N}_{0}$ with $k+l=n-2$ and $(p, q) \in \mathbb{N}_{0} \times \mathbb{N}$ (sic!) with $p+q=s-1$, the Legendrian knot $L$ shown in Figure [6 is an exceptional realisation of $T(s,-(s n-1))$ in $S^{3}$ with $\mathrm{tb}=-s(s n-1)$.

Proof. For the topological aspect of the statement, see the Kirby moves in Figure 7 The effect of the final $1+n$ handle slides is now shown in Figure 8 . Before, we explained that the cancellation of the two surgeries (after the handle slides) can be interpreted as a slam-dunk of the 0-framed meridian, which turns the $(-n)$ framed unknot into an unknot with surgery framing $-n-1 / 0=\infty$. Alternatively, we can actually interpret the complete move (handle slides and cancellation of the surgeries) as a slam-dunk of the $(-n)$-framed meridian to the 0 -framed unknot. This turns the latter into an unknot with framing $0-1 /(-n)=1 / n$, and leaves $L$ unchanged. The $(1 / n)$-surgery along the unknot is equivalent to removing a tubular neighbourhood of the unknot, twisting the neck $-n$ times, and then regluing the 


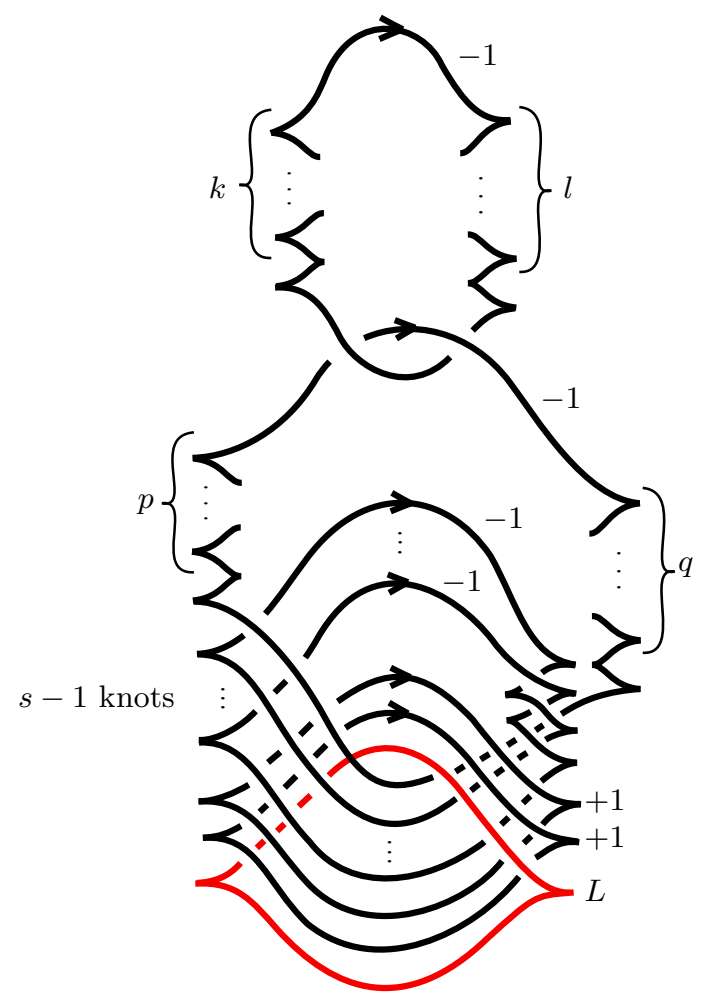

FIGURE 6. Exceptional realisations of $T(s,-(s n-1))$.

solid torus with the identity map. This results in the diagram on the right of Figure 8 ,

The linking matrix, which we need for the computation of various invariants, is now

$$
M=\left(\begin{array}{cccccccc}
0 & -1 & \overbrace{-1}-1 & \ldots & -1 & -1 & 0 \\
-1 & 0 & -1 & -1 & \ldots & -1 & -1 & 0 \\
-1 & -1 & -3 & -2 & \ldots & -2 & -1 & 0 \\
-1 & -1 & -2 & -3 & \ldots & -2 & -1 & 0 \\
\vdots & \vdots & \vdots & \vdots & \ddots & \vdots & \vdots & \vdots \\
-1 & -1 & -2 & -2 & \ldots & -3 & -1 & 0 \\
-1 & -1 & -1 & -1 & \ldots & -1 & -s-1 & -1 \\
0 & 0 & 0 & 0 & \ldots & 0 & -1 & -n
\end{array}\right) .
$$

In order to verify that $L$ is exceptional, we compute the $d_{3}$-invariant of the contact structure on $S^{3}$ described by the surgery diagram in Figure 6. The number of 2 -handles in this Kirby diagram is $s+3$, so the Euler characteristic of the handlebody is $\chi=4+s$. The signature is $\sigma=-1-(s-2)=1-s$ since, compared with with the diagram in Figure 5 we have $s-2$ additional (-1)-framed unknots from the blow-downs shown in Figure 7 . 

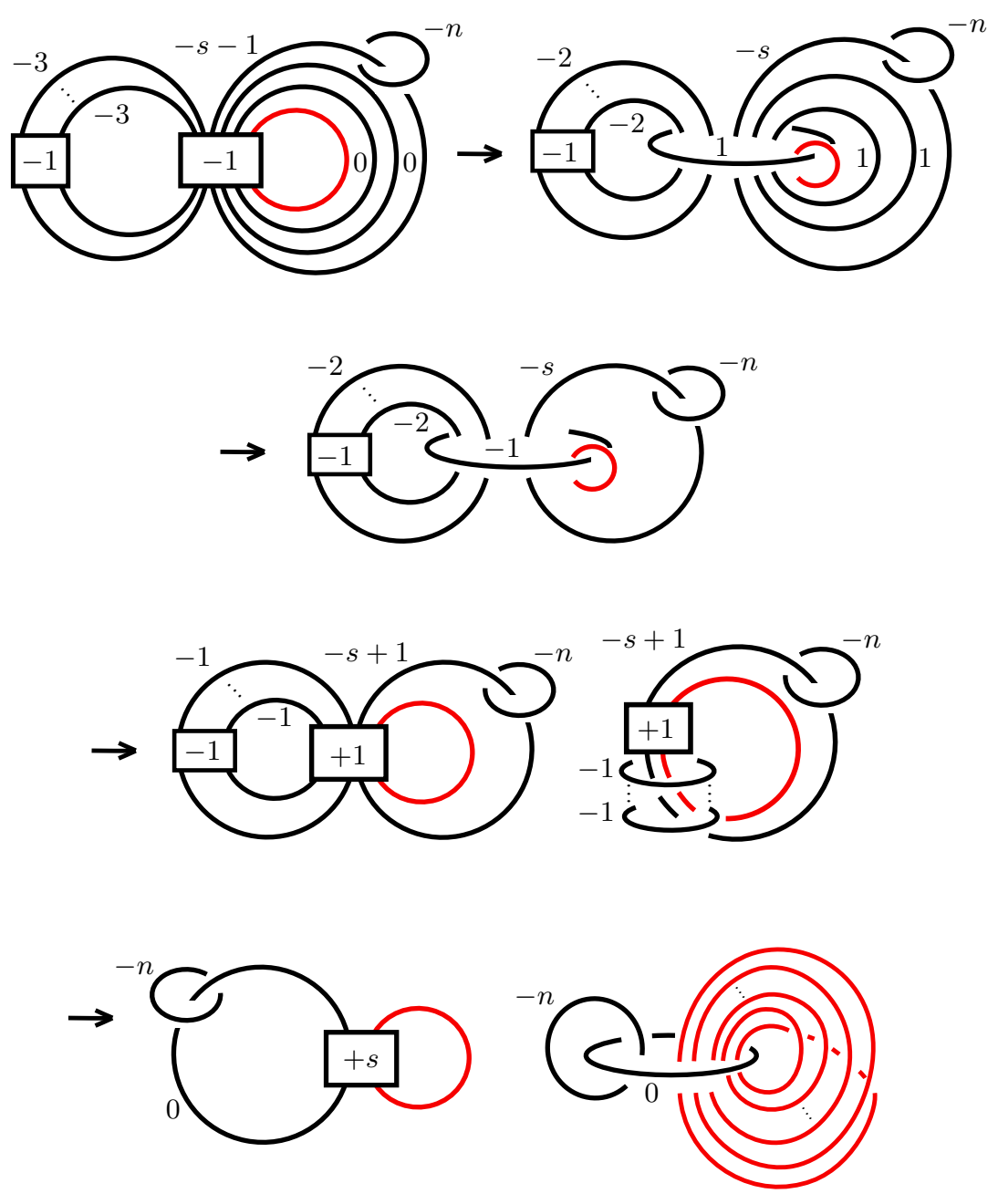

Figure 7. Kirby moves for Figure 6.

The vector of rotation numbers is

$$
\underline{\mathrm{rot}}=(0,0, \underbrace{1, \ldots, 1}_{s-1}, q-p, l-k)^{\mathrm{t}},
$$

and the solution $\mathbf{x}$ of $M \mathbf{x}=\underline{\text { rot }}$ is given by

$$
(-1-s u,-1-s u, \underbrace{u, \ldots, u}_{s-1}, u+1,-2 q)^{\mathrm{t}},
$$

where $u:=k-l+2 q n-1$. It follows that

$$
c^{2}=\mathbf{x}^{\mathrm{t}} M \mathbf{x}=4 n q^{2}+4 q(k-l)-s+1,
$$

and

$$
d_{3}=n q^{2}+q(k-l)-\frac{1}{2}
$$



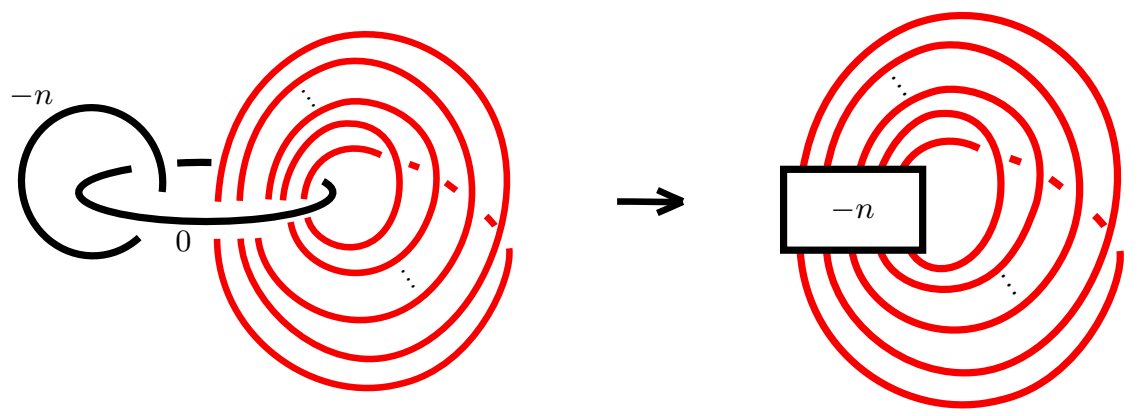

Figure 8. The final handle slides as a slam-dunk.

For $q \geq 1$, as assumed in the proposition, we have $d_{3}>-1 / 2=d_{3}\left(\xi_{\text {st }}\right)$, so this diagram does indeed define an overtwisted contact structure on $S^{3}$.

For the computation of $\operatorname{tb}(L)$ in the surgered $S^{3}$, one determines the determinants of $M$ and the extended matrix $M_{0}$ by a simple row reduction. One finds $\operatorname{det} M=(-1)^{s-1}$ and $\operatorname{det} M_{0}=(-1)^{s-1}(1-s(s n-1))$. The formula from Section 3 then yields $\operatorname{tb}(L)=-s(s n-1)$.

Remark 5.3. Analogous to Remark 3.1 one can alternatively compute $\operatorname{tb}(L)$ from the effects of the Kirby moves on the framing given by a Legendrian push-off $L^{\prime}$ of $L$ in the original diagram. After the moves in Figure 7, we have $\operatorname{lk}\left(L, L^{\prime}\right)=s$ (as knots in the unsurgered $S^{3}$ ). After the slam-dunk in Figure $8 s$ strands of the parallel knot $L^{\prime}$ receive a $(-n)$-twist relative to each of the $s$ strands of $L$, so we arrive at $\operatorname{tb}(L)=s-n s^{2}=-s(s n-1)$.

The final lemma tells us that the tight contact structures on $L\left(n s^{2}-s+1, s^{2}\right)$ obtained by Legendrian surgery on these $(s-1)(n-1)$ Legendrian realisations of $T(s,-(s n-1))$ in $S^{3}$ can be distinguished from one another - and from the ones coming from Proposition 5.1 - by their Euler class. This completes the proof of Theorem 1.1.

Lemma 5.4. The Euler class of the tight contact structure $\xi=\xi_{k, l, p, q}$ on the lens space $L\left(n s^{2}-s+1, s^{2}\right)$ obtained by surgery on the link in Figure 6 , including a contact (-1)-surgery along $L$, is

$$
e(\xi)=(p-q+1) n s+(l-k) s \bmod n s^{2}-s+1 .
$$

Remark 5.5. Notice that $l-k$ takes values in the range

$$
l-k \in\{-n+2,-n+4, \ldots, n-4, n-2\} ;
$$

for $p-q+1$ the range is

$$
\{-s+2,-s+4, \ldots, s-4, s-2\} \text {. }
$$

So the first summand in the expression $e(k, l, p, q)$ for $e(\xi)$ varies in steps of size $2 n s$, whereas the second summand ranges between $\pm(n-2) s$. This means that there are no duplications in this list of Euler numbers, at least before reducing modulo $n s^{2}-s+1$.

In $\mathbb{Z}$ we have

$$
e_{\min }:=-n s^{2}+(n+2) s \leq e(k, l, p, q) \leq n s^{2}-(n+2) s=: e_{\max },
$$


so $e_{\max }-e_{\min }<2\left(n s^{2}-s+1\right)$. When we bring the negative $e(k, l, p, q)$ modulo $n s^{2}-s+1$ into the range $\left(0, n s^{2}-s+1\right)$, they are congruent to 1 modulo $s$, whereas the positive $e(k, l, p, q)$ are divisible by $s$, so there are no duplications even modulo $n s^{2}-s+1$. Moreover, we have

$$
e_{\min }+n s^{2}-s+1=(n+1) s+1>(n-1) s-1
$$

and

$$
-(n-1) s+1+n s^{2}-s+1=n s^{2}-n s+2>e_{\max },
$$

which implies that there are also no duplications with the Euler numbers (modulo $n s^{2}-s+1$ ) coming from Proposition 5.1.

Proof of Lemma 5.4. We label the meridional classes corresponding to the knots in Figure 6 from bottom to top as

$$
\left[\mu_{L}\right],\left[\mu_{1}\right],\left[\mu_{2}\right],\left[\nu_{1}\right], \ldots,\left[\nu_{s-1}\right],\left[\alpha_{s}\right],\left[\beta_{n}\right] .
$$

We then compute the relations between these generators from the matrix $M$ as in Section 4 to obtain

$$
\begin{gathered}
{\left[\mu_{1}\right]=-\left[\mu_{L}\right], \quad\left[\mu_{2}\right]=-\left[\mu_{L}\right], \quad\left[\nu_{1}\right]=\ldots=\left[\nu_{s-1}\right]=(1-n s)\left[\mu_{L}\right],} \\
{\left[\alpha_{s}\right]=-n s\left[\mu_{L}\right], \quad\left[\beta_{n}\right]=s\left[\mu_{L}\right], \quad\left(n s^{2}-s+1\right)\left[\mu_{L}\right]=0 .}
\end{gathered}
$$

From the vector rot we then compute

$$
\begin{aligned}
e(\xi) & =\left[\nu_{1}\right]+\cdots+\left[\nu_{s-1}\right]+(q-p)\left[\alpha_{s}\right]+(l-k)\left[\beta_{n}\right] \\
& =((p-q+1) n s+(l-k) s)\left[\mu_{L}\right]
\end{aligned}
$$

as claimed.

Remark 5.6. In the proofs of Propositions 4.2 and 5.2 we used the $d_{3}$-invariant to show that $L$ lives in an overtwisted contact structure on $S^{3}$, and hence $L$ is exceptional, since Legendrian surgery along $L$ produces a tight contact structure. The information that $L$ is exceptional was not actually necessary for proving Theorem 1.1. Rather, the converse is true: the fact that the resulting tight contact structure on $L\left(n s^{2}-s+1, s^{2}\right)$ is different from any structure obtained from a Legendrian realisation of $T(s,-(s n-1))$ in $\left(S^{3}, \xi_{\text {st }}\right)$ gives an alternative criterion for establishing the exceptional character of $L$.

Acknowledgements. The research for this paper was done during an enjoyable Research in Pairs stay at the Mathematisches Forschungsinstitut Oberwolfach, 27 March - 9 April 2016. We thank the Forschungsinstitut for its support, and its efficient and friendly staff for creating, once again, an inspiring environment.

S.O. is partially supported by the grants HÜBAP FBB-2016-9429 and TÜBİTAK \#115F519.

\section{REFERENCES}

[1] J. Bailey And D. Rolfsen, An unexpected surgery construction of a lens space, Pacific J. Math. 71 (1977), 295-298.

[2] M. Culler, C. McA. Gordon, J. Luecke and P. B. Shalen, Dehn surgery on knots, Ann. of Math. (2) 125 (1987), 237-300.

[3] F. Ding and H. Geiges, A Legendrian surgery presentation of contact 3-manifolds, Math. Proc. Cambridge Philos. Soc. 136 (2004), 583-598.

[4] F. Ding, H. Geiges and A. I. Stipsicz, Surgery diagrams for contact 3-manifolds, Turkish J. Math. 28 (2004), 41-74. 
[5] S. Durst And M. Kegel, Computing rotation and self-linking numbers in contact surgery diagrams, arXiv: 1605.00795.

[6] J. B. Etnyre and K. Honda, Knots and contact geometry I: Torus knots and the figure eight knot, J. Symplectic Geom. 1 (2001), 63-120.

[7] H. Geiges, An Introduction to Contact Topology, Cambridge Stud. Adv. Math. 109 (Cambridge University Press, Cambridge, 2008).

[8] H. Geiges And S. Onaran, Legendrian rational unknots in lens spaces, J. Symplectic Geom. 13 (2015), 17-50.

[9] E. Giroux, Structures de contact en dimension trois et bifurcations des feuilletages de surfaces, Invent. Math. 141 (2000), 615-689.

[10] R. E. Gompf, Handlebody construction of Stein surfaces, Ann. of Math. (2) 148 (1998), 619-693.

[11] R. E. Gompf And A. I. Stipsicz, 4-Manifolds and Kirby Calculus, Grad. Stud. Math. 20 (American Mathematical Society, Providence, RI, 1999).

[12] M. Hedden, On Floer homology and the Berge conjecture on knots admitting lens space surgeries, Trans. Amer. Math. Soc. 363 (2011), 949-968.

[13] K. Honda, On the classification of tight contact structures I, Geom. Topol. 4 (2000), 309-368.

[14] P. Kronheimer, T. Mrowka, P. Ozsváth and Z. Szabó, Monopoles and lens space surgeries, Ann. of Math. (2) 165 (2007), 457-546.

[15] P. Lisca and G. Matić, Tight contact structures and Seiberg-Witten invariants, Invent. Math. 129 (1997), 509-525.

[16] P. Lisca, P. Ozsváth, A. I. Stipsicz and Z. Szabó, Heegaard Floer invariants of Legendrian knots in contact three-manifolds, J. Eur. Math. Soc. (JEMS) 11 (2009), 1307-1363.

[17] L. Moser, Elementary surgery along a torus knot, Pacific J. Math. 38 (1971), 737-745.

[18] P. Ozsváth and Z. Szabó, On knot Floer homology and lens space surgeries, Topology 44 (2005), 1281-1300.

[19] O. Plamenevskaya, On Legendrian surgeries between lens spaces, J. Symplectic Geom. 10 (2012), 165-181.

[20] J. Rasmussen, Lens space surgeries and L-space homology spheres, arXiv:0710.2531.

[21] M. TANGe And Y. YAMADA, Four-dimensional manifolds constructed by lens space surgeries along torus knots, J. Knot Theory Ramifications 21 (2012), 1250111, 65 pp.

Mathematisches Institut, Universität ZU KÖln, Weyertal 86-90, 50931 Köln, GerMANY

E-mail address: geiges@math.uni-koeln.de

Department of Mathematics, Hacettepe University, 06800 Beytepe-Ankara, Turkey

E-mail address: sonaran@hacettepe.edu.tr 